\title{
Kapitalistische Stadtplanung und die Illusion demokratischer Bürgerinitiative
}

Der vorliegende Aufsatz ist im Zusammenhang der Diskussion uber Einzelprobleme sozialstaotlicher Intervention entstanden, deren erstes Ergebnis die einleitende Arbeir von Wolfgang Muller und Christel Neusis 3 "Die Sozialstaatsillusion und der Widerspruch von Lohnarbeit und Kapital" ist. Die dort allgemein entwickelte Kritik wird hier an einem Punkt ins einzelne verfolgt: der Offeschen These von den "horizontalen Disparitaten". (1).

Diese These soll hier kritisiert werden als Beispiel für an den Erscheinungsformen gesellschaftlicher Benachteiligung orientierte Konzeptionen der schrittweisen und arbeitsteiligen Beseitigung "sozialer Mißstånde", wie sie etwo von den Jungsozia.. listen vertreten werden und in der Stadtteilarbeit vieler linker Gruppen ihren Ausdruck finden. (2) Direkt auf die Disparitätenthese beziehen sich besonders Gruppen, die von der beruflichen Aufgabe her einer sozial engagierten Praxis verpflichtet sind und die gerade in der Begrifflichkeit der Disparitätenthese eine theore tische Überhöhung ihrer eigenen Praxis finden können, wie Sozialarbeiter, Architekłen, Stadtplaner, Pädagogen, Psychologen etc. Sie können darüberhinaus die von Offe angegebenen praktischen Schlußfolgerungen aus jener These als konkrete Handlungsanweisung verstehen: Der "professionalisierten Intelligenz" wird eine exponierfe Bedeutung zugemessen als Initialzuindung für sich außerhalb des traditionellen Instifutionensystems bildende "Basisgruppen" disparitär benachteiligter Bürger, die ols Motor einer "revolutionären Transformation" interpretiert werden.

Nachdem seit geraumer Zeit diverse Gruppen eine derartige Konzeption praktisch erproben, ist es notwendig, exemplarische Erfahrungen dieser Versuche den theoretischen Konstruktionen der Disparifatenthese gegenüberzustellen und damit die bei Muiller und Neusiuß entwickelte Kritik empirisch zu belegen.

Rekapiłulieren wir zunåchst die Grundthesen der Disparitåtentheorie: Ausgangspunkt von Offes Analyse ist die These, daß den staatlich regulierten Spätkapitalismus drei fundamentale Systemprobleme beherrschen, deren Bewältigung sich für das staatliche Handeln zum "interessenunspezifischen, 'sachgesetzlichen' Imperativ" verselbståndigta sokonomische Stabilität, außerwirtschaftliche und milirärpolitische Beziehungen und die Sicherung der Massenloyalität (3). Daraus schließt Offe einmal auf eine "Lockerung der Beziehung zwischen Arbeitsleistung und Einkommen", da das Einkommen zunehmend politischen Regulationen unterworfen werde und die Form "angemessener Entschädigung" anzunehmen tendiere; zum andern lasse sich eine "Lockerung der Beziehung zwischen Einkommen und Le- 
benschancen" feststellen. Denn die Beschränkung staatlichen Handelns auf ein umsichtiges crisis management der langfristigen Vermeidungsstrategie erzeuge ein "konzentrisches Prioritätenschema staatlichen Eingreifens von gesellschaftlichen Bedürfnissen und Problemberei chen, in dem diese umso näher beim Zentrum der höchsten Dringlichkeitsstufe stehen, je mehr die Verletzung entsprechender Ansprüche ökonomische Stabilitätsvoraussetzungen infrage stellen würde" (4). An der Peripherie lägen diejenigen gesellschaftlichen Bedürfnisse, die ihre Berücksichtigung nicht durch Konfliktdrohung erzwingen könnten; dagegen besåßen diejenigen Interessengruppen und Problembereiche eine strukturelle Privilegierung, die eine funktionale Unentbehrlichkeit vorweisen könnten. Somit sei der Pauperismus des 19. Jahrhunderts abgelöst durch den Pauperismus der depressed areas in der Unterversorgung der alle Bürger betreffenden Bereiche wie Bildung, Verkehr, Wohnung, Gesundheit; die gleiche Benachteiligung gelte aber auch für "biographische Marginalsituationen" wie Vorschule, Alter, Arbeitslosigkeit, Geisteskrankheit, Kriminalität und für "gesellschaftliche Ausnahmesituationen ethnischer Minderheiten, zukunftsloser Wirtschaftszweige, der Slums und der strukturellen Armutsgebiete".

Die gesellschaftliche Benachteiligung betrifft danach also einmal "Situationsgruppen", denen jeder Bürger mit Teilen seiner Existenz angehört, zum anderen "Randgruppen", die aus dem Produktionsprozeß Herausgefallene umfassen. Beiden gemeinsam sei die Unterdrückung bestimmter Bedürfnisse, deren besonderer Charakter darin liege, daß sie "nicht an die herkömmliche Dimension der Tauschund Leistungsgerechtigkeit und traditionalistischer Statusansprïche gebunden sind" (6). Deshalb könnten sie "neue Formen der sozialen Interaktion und neue Formen der Bedürfnisbefriedigung, kurz: historischen Fortschritt herbeiführen" , wenn es gelänge, sie in Institutionen solidarischer Willensbildung zum Ausdruck kommen zu lassen.

In den "Situationsgruppen" glaubt Offe also, ein neues "gesellschaftliches Subjekt historischen Wandels" gefunden zu haben. Die systemumwälzenden Perspektiven von deren Mobilisierung theoretisch plausibel zu machen, bereitet ihm allerdings nicht unerhebliche Schwierigkeiten. Es bedürfe dazu neuer Aktions- und Organisationsformen, deren Erfolg davon abhinge, ob es gelänge, der kollektiven Deprivation der Wirklichkeit eine anschauliche andere Moglichkeit gegenuberzustellen. Dazu müsse die professionalisierte Intelligenz initiierende Funktiow onen übernehmen: "Das Personal der zurückbleibenden Institutionen des Bildungs= wesens, des Gesundheitswesens, der Stadtplanung und der Architektur und einiger weiterer Bereiche in Technologie, Administration, Planung und Forschung ist zumindest von seinen spezialisierten Berufskenntnissen her in der Lage, den Konflikt zwischen Wirklichkeit und Moglichkeit für sich und andere zu konkretisieren und so in einem politischen Bezugsrahmen systemtranszendierende Bedürfnisinterpretationen anzubieten, die freilich nur auf der Basis neuer politischer Organisationsformen außerhalb des politischen Systems folgenreich werden könnten" (ク). Auf diese Weise könnte die fast unüberwindliche Starrheit des 
politischen Institutionensystems in einem Demokratisierungsprozeß von unten, durch organisierte Bedurfnisartikulation in den peripheren Bereichen in Bewegung gebracht und durch die Konfliktdrohung das "Zentrum" zum ausgleichenden Eingreifen gezwungen werden.

\section{DIE KONKRETISIERUNG DER DISPARITÅTENTHESE FÜR DIE STADTPLANER}

Wie man sich den "gesellschaftlichen Wandel" durch die Mobilisierung von Sifuationsgruppen vorzustellen hat, hat Offe in einem Vortrag bei einem Kolloquium des Münchener Instituts für Städtebau und Landesplanung (8) für die Berufspraxis von Architekten, Stadt- und Regionalplanern konkretisiert. Diese Ausführungen, die über die Planungsdisziplinen hinous wenig bekannt geworden sind, verdienen deshalb besonderes Interesse, weil Offe hier aus der Diskussion technokratischer Entscheidungsmodelle als praktisch-politische Konsequenz der Disparitätenthese eine Konzeption planungsbezogener $p \circ \mid i-$ is eher Basisorganisationen entwickelte.

Ausgehend von der Frage nach der Mrglichkeit, die Bedingungen zukünftigen Lebens mit den Bedurrfnissen in Einklang zu bringen, werden die Bereiche des Bildungs- und Gesundheitswesens und der Stadtplanung begriffen als solche, in denen wegen ihrer relativen Ferne von den zentralen Bereichen Wirtschaft, Militår und Politik die Entscheidungen sich in einem gewissen Spielraum bewegen könnten, weil auf ihnen nicht der unmittelbare Druck der Systemerhalitung laste. Dieser Spielraum könne erweitert und für eine bedürfnisgerechtere Planung genutz" werden durch die Mobilisierung der Betroffenen, die innerhalb von Organs ationen außerhalb des etablierten Institutionensystems- sich ihrer Beduirfnisse in einem kollektiven Willensbildungsprozeß bewußt werden und sie artikulieren sollten. Denn die in diesen peripheren Bereichen auftretenden Sachzwänge wie die institutionellen (genannt werden Verwal fungsstruktur, Finanzierungsmơglichkeiten, Eigentumsordnung, sowie das Fehlen von zentralen Planungsinstitutionen), die Zwänge der knappen Zeit und die der knappen Mittel sind nach Offe nur die "Folge eines institurionellen Defekts, denn Sachzwang herrscht dort, wo die Institutionen der Öffentlichkeit vertrocknet sind, bzw. wo es nicht gelungen ist, sie durch neue politische Institutionen zu arsetzen ${ }^{\text {BI }}$ (9). Fine von der Basis her" wiederbelebte Öffentlichkeit aber kBnne die dem Entscheidungsspielraum durch "Sachgesetzlichkeit" gezogene Grenze immer wieder hinausschieben. "Diese Grenze bildet eine Barriere, die prinzipiell auflesbar, nur jeweils noch nicht aufgelost ist" (10). 
Eine $n$ ur am Sachawang orientierte technokratische Reformpolitk kritisierte Offe als unzureichend: Es bedurfe in Bereichen wie Arbeitsmarkt-" Verkehrs-, Bildungs-, Gesundheits- und Wohnungsbcupolitik zur Lossung der Probleme "der aurgeklarten Kooperctionsbereitschaft der Bevslkerung ${ }^{\text {is }}$. Die traditionellen Mit Pel, materielle Anreize und Bestrafungen, reichten nicht aus, "auf einem relativ hohen Niveau dep skononischen Prosperifait und im Rahmen gesicherter Grundrechie ... komplemenicires Verhalten zu eizeugen .

Solche cufgek lïrte Kooperationsbereitschaft, mit deren Enistehen uberkommene Verhaltensmuster und Gesellschaftsbilder jedenfalls zum Teil verfluss igr würden, gedeihe aber prinzipiell nus unrer Bedingungen, die den Bürgern die freie Artim kulation ihrer Bedürnisse und die entsprechenden politischen Handlungsspielpơume zur Verfugung stellen.

An dieser Kritik der technokratischen Reformpolitik wird Offes Vorstellung von dem Verlauf der gesellschaftlichen Umwailzung deutlich: Das angebotene orgonisatorische Konzept dep "Situctionsgruppen" dient zuncichst dazu, technokrotische Reformpolitik um jene cufklärerische Komponente zu bereichern, die es thr erst ermoglicht, ous dem Dilemma ihrer eigenen Sabotage herauszukommen, denn "das apothische Bewußtsein entpolitisierter Verwoltungsobjekte, das von rechnokrarischen Entscheidungsstrukturen produziert wird, ist zugleich deren gravierendes Funktionshindernis". (11) Die außerhalb der Institutionen organisierten Gruppen sollen also zunächst den reibungsloseren Ablauf notwendiger struktu reller Veränderungen gewährleisten. Der Entscheildungsspielraum, der diesen Gruppen explizit gemacht werden muß, um ihr "Mitmachen" anzuregen, führt dann aber "mit erfreulicher Folgerichtigkeir" dariberhinaus, wie Offe meint, denn die

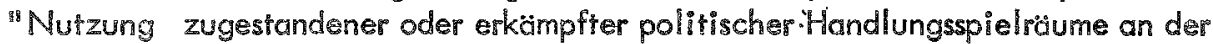
Basis (tendiert) dahin, die beiden Systemprämissen opolitische Repräsentation und privatwirtschaftlicher Invastitionsentscheidung- zu siören oder zu zerstören" . (12)

Auf dem Hintergrund dieser vagen Einschätzung einer mogglichen gesellschaftlichen Verïnderung kritisiert Offe verschiedene Vorschläge und praktizierteVersuche von Stadiplanern, die Bedirfnisse von Planungsbetroffenen in den Planunggs-prozeß einzubeziehen, die er in einer Folge von vier Stufen darstellt, worin jem de Stufe die Schwierigkeiten der vorangegangenen uberwunden habe.

Die ersten beiden Stufen behandeln die Ergänzug der Arbeit der Planer zunächst durch interdisziplinäre Kooperation (Stufe I), dann durch empirischmsoziologische Methoden der Befragung (Stufe 2). Hier formuliert Offe als grundlegenden Einwand: " Es gehsirt zum Merkmal gesellschaftlicher Bedurfnisse und Interessen, daß sie nicht vom isolierten Individuum, das isoliert von konkreten Lebenssituationen befragt wird, angegeben werden ksnnen, sondern daß sie nur in einer kollektiven Kommunikation, und zwar in unmitrelbarer Năhe zu den Situationen, ubber die diskutiert wird, aufzudecken sind" 
Als dritte Stufe wird ein Modell genanni, das die Schwierigkeiten einer sich ous das herksmmliche Methodenarsenal ges ellschafis- und planungswissenschaftlicher Disziplinen beschrönkenden Vorgehensweise berucksichrigt: die schon klassische Anwaltsplanung, wie sie Poul Dovidoff beschrieben hat (14), der, ausgehend ven einem pluralistischen Demokratieverstandnis, auch die Teilnahme von bisher noch nicht ins etablierte System von Interessenvertrefung inbezogenen Gruppen am Planungsprozeß erwirken will. Um den Gefahren eines der Öffentlichkeit entzo * genen burrokratischen Managements zu begegnen, schlägr Davidoff vor, den Widerstreit von Interessen offentlich auszutrogen, indem jede Inieressengruppe un ter Mitwirkung eines Anweltsploners einen von ihr mit Nachdruck zu vertreten w den Entwicklungsplan für die Kommune aufstellt. Als Anwalt habe der Spezialish die Sprache der Planung transparent zu machen auf die in einzelnen Planen zur Geltung kommenden Interessen, - nicht denunziatorisch, sondern Fakten und Giunde abwägend, die fü die einzelnen Vorschläge sprechen und andere wer ger winschenswert machen.

Diese Konzeption bezichtigt Offe "offensichtlicher Naivirot", da nicht cngenom men werden konne, daß sich die Planungsbehorde in der Rolle eines neutralen Richters befinde, der ohne Eigeninteresse die kontroversen Vorschläge abwigit und Lossungen finder, noch ksnne devon ausgegeangen werden, daß alle beteiligm ten Interessengruppen so weit organisiert seien daß sie die Gemeinsamk it threr Bedürfnisse und deren Inhalte erkennen könnten. (15)

Das 4. und letzte Modell der Stufenfolge, das die Beschrönkungen der Anweltyplanung in "radikaler Konsequenz durchbricht" sieht folgendermaßsen aus: "Wenn das Mirspracherecht bei einem plurelistischen Planungsprozeß auf wenige, konsolidierte Interessenorganisationen beschränkt ist: wenn demzufolge zahlreiche porentielle Interessengruppen vom Mitspracherechr̂ ausgeschlossen sind und desw halb keine Chance haben, sich als Tråger kollektiver Beduirfnisse zu ctablieren, und deshalb in der schicksalshörigen Apathie derer beharren, die allenfalls Schlimmeres zu erwarten haben; wenn außerdem die Unparteilichkeit derjenigen Entscheidungsinstanz, die im pluralistischen Kräfrespi schließlich den Richtspruch fullt, durchous in Zweifel gezogen werden meP: dann ist es nur konsequent, wenn die Planungsinstanzen ihre Orientierungen von planungsbezogenen politischen Basisorganisationen beziehen, die sie freilich selbstas ins Leben zu rufen hät ten ${ }^{\text {a }}$. (16)

Gerade dies letzre Konzept kommi dem latenten Wünschen der Mirglieder der In telligenz in den Planungsbereichen sehr enrgegen. Auf der Grundlage der zentralen Positionen - 1. der These von der relativen Entferntheit von Bereichen wie Siogdtebau, Verkehr, Bildung, Gesundheit ete, von den zeniralen Bereichen Wirtschost und Politik, und 2. der These vom Spielraum für wesentliche Reformen in diesen Bereichen werden Handlungsspielräume für die Planerintelligenz unterstellt zur Beeinflussung von Planungen im Interesse der Bepoanten. Es ist deher kein Zufall. wenn einer der Kronzeugen, die Offe fur sein viertes Modell anfuhrt, die Basisgruppe Kreuzberg, die hauptsichlich ous Architekiturstudenten bestand, ganz naturwichsig auf der Basis eines derartigen ("Offeschen") Ansatzes arbeitet. Wegen ihrer immer noch latenten Relevenz für die Praxis von Stadtrell wruppen mussen Offes grundlegende Thesen im folgenden untersucht werden. 


\section{DIE KONZERNINTERESSEN AN DER REGIONALEN NEUORDNUNG UND DIE STRATEGIE LOKALER BASISORGANISATION}

Aus der Vielzahl der US-amerikanischen Versuche von Gemeinde-Selbstbestimmung in der Stadrplanung wollen wir die Erfahrungen einer organisatorisch äusserst erfolgreichen Gruppe in San Francisco untersuchen, um zu zeigen, daß selbst unter diesen extrem günstigen Bedingungen die. These von der Entferntheit der disparitären Lebensbereiche gegenüber dem zentralen Bereich Wirtschaft sich nicht aufrechterhalten laßst daß vielmehr diese Bereiche als Teile der Infrastruktur nach den Verwertungsinteressen des Kapitals umstrukturiert werden und von daher der Entscheidungsspielraum von Community Power auf marginale Fragen beschränkt ist.

Am Beispiel von Entwicklungen in der Region von San Francisco lassen sich die Umstrukturierung der Infrastruktur und die damit verbundene Zentralisierung von Wirtschaft und Verwaltung unter der Führung der Konzerne verfolgen. Diese Tendenzen können sich im Gebiet der Bucht von San Francisco ohne Behinderung durch die Interessen partikularer Kapitalfraktionen entfalten, da den Neverern keine alteingesessene Oligarchie gegenübersteht. Der Handelskammer von San Francisco zufolge, "baut die Wirtschaft von San Francisco auf ihrer Rolle als Hauptverwaltungszentrum des amerikanischen Westens auf". Diese basiert auf der Konzentrátion von Banken, Versicherungen und Immobiliengesellschaften. Die aufstrebenden Industriestädte im Gebiet der Bucht wie Oakland, Hayward, oder San Jose sind ihrerseits an der Entwicklung des Handels- und Verwaltungszentrums von San Francisco interessiert, denn "sie glauben, daß (die Entwicklung ihrer Indusirie) von einem starken Finanzzentrum und einer wirkungsvollen regionalen Koordination abhångt" . (17) Das heißt, die spezifische Art der bereits in San Francisco und der weiteren Region vorgenommenen Infrastruktur-Investitionen haben die Interessen von Industrie- und Bankkapital in einer Entwicklungsplanung für die Region vereint, die San Francisco als Mittelpunkt von Handel und Verwaltung ausbauen soll.

Das Gebiet der Bucht von San Francisco und San Pablo, das gegenwärtig über 90 Gemeinden mit ca. vier Millionen Einwohnern umfaßt und in neun Verwaltungsbezirke unterteilt ist, hat in den letzten zwanzig Jahren einen starken industriellen Aufschwung genommen, der sich Trendprognosen zufolge fortsetzen wird: Bis 1980 werden voraussichtlich sieben Millionen Menschen im Baygebiet leben. Wie die Konzerninteressen die von dieser Entwicklung geforderte Neustrukturierung und Erweiterung der Infrastruktur bestimmen, haben Beagle, Haber und Wellman dokumentiert. (17) Die Konzerninitiativen verlaufen in drei programmatischen Stoßrichtungen: $a$. regionale Vereinheitlichung der Verwaltung, $b$. Errichtung eines regionalen Schnellverkehrssystems, c. Stadtsanierung in den Ballungszentren. 
Die grundlegende Zielsetzung ist die Vereinheitlichung der Verwaltung ouf regionaler Ebene, die von der seit dem Ende des 2. Weltkrieges arbeitenden Planungsgruppe BAY AREA COUNCIL verfolgt wird, einer Superlobby, die sich aus den leitenden Angestellten der größten Konzerne des Baygebietes zusammensetzt und fi nanzielle Unterstützung von allen neun Bezirksverwaltungen erhält. Ihr Programm lautet, "das Gebiet der Bucht von San Francisco auf einer politischen Basis zu organisieren, die der wirtschaftlichen Realität entspricht" wie es ein Vizepräsident des COUNCIL formulierte. Dies besagt, die politischen Institutionen sollen als Instrument der ungehinderten Durchsetzung der Interessen der größten Konzerne mo= dernisiert werden, da für sie ein einheitlicher und zentrolisierter Markt erforderlich ist. Daß damit auch der Beteiligung der Öffentlichkeit an der lokalen Verwaltung ein Ende gesetzt wird, stellte eine nationale Vereinigung der größten Konzerne, das COMMITTEE FOR ECONOMIC DEVEL OPMENT klar. Wie das COMMITTEE

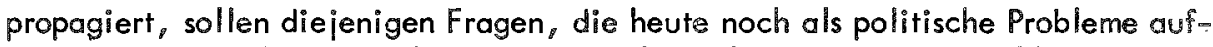
gefaßt werden, als technische Aufgaben behandelt werden, um Konflikte und Ineffizienz zu vermeiden. Das COMMITTEE FOR ECONOMIC DEVELOPMENT betreibt diese Strategie dadurch, daß es die Bildung gutausgerüsteter und mächtiger Regionalverwaltungen unterstützt, wodurch die lokalen Verwaltungseinheiten um $80 \%$ reduziert werden sollen. Weiter sollen nach Vorstellung des COMMITTEEs die Verfassungen der Bundesstaaten dahingehend geändert werden, daß die Vielzohl von kommunalen Wahlen stark verringert und Selbstverwaltung nur'noch denen zugestanden wird, "die sie handhaben können" (18).

Der zweite Komplex, mit dem sich die technokratischen Planungsinitiativen der Konzerne in der Region von San Francisco befassen, ist die Errichtung des Schnellverkehrssystems BAY AREA RAPID TRANSIT (BART), das das Stadtzentrum mit Konsumenten und Arbeitskräften der gesamten Region verbinden soll. Dieses Schnellverkehrssystem soll einmal zur Überwindung der vom Auto verursachten Verstopfung der Innenstadł dienen und dadurch den Unternehmen im Stadtzentrum Wachstum und neue Prosperität garantieren. Zum anderen soll es mehrere, bisher nur lose zUsammenhängende Märkte von Arbeitskräften, Waren und Dienstleistungen mit einander verknüpfen. Allerdings trifft die Trassenführung eine sorgföltige Auswahl: mit der Innenstadt verbunden werden lediglich die wohlhabenderen Vororte. (19) Dies erschließt den in der City ansässigen Unternehmen das white-collar-Arbeitskräftepotential der gesamten Region. Ebenso wird damit den anspruchsvolleren, d.h. zahlungsföhigeren Konsumenten leichter Zugang zu den exklusiven Einzelhandelsgeschäften des Citybereichs und den ihm eigenen speziellen Dienstleistungen verschafft. Die Linien verbinden aber nicht die Innenstadt, in der die Arbei w terviertel liegen, mit dem industriellen Hinterland; berwhren oder durchschneiden sie die innerstädtischen Ghettos wie z. B. die Oakland Ghettos, so gibt es dort keine Station. Das BAY AREA RAPID TRANSIT SYSTEM ist also nicht darauf angelegt, Arbeitern aus den schwarzen und braunen Ghettos Arbeitsplätze in der Industrie außerhalb der City zu erschliessen. Es leistet somit nicht einmal als Ne- 
beneffekt einen Beitrag dazu, den wenig qualifizierten, beschäfrigungslosen Arbeitern Zugang zu einem grßßeren, regionalen Angebot von Arbeitsplätzen zu verschaffen.

Von Anfong an war dos BAY AREA RAPID TRANSIT SYSTEM als ein offentliches Verkehrsmitrel mit einem beschränkten Benutzerkreis geplant worden. Sein Einzugsbereich wurde definiert durch die klar erkennbare Zielsetzung, die Zusammenfassung von Bank- und Handelskapital sowie von Verwaliungsfunktionen von Industriekonzernen in San Francisco voranzurreiben. Die Folge davon ist eine star$k$ innerstadtische Verdichung, ablesbar bereits jetzt an einem enormen Bauboom. Damit bewirkt das Schnellbahnsystem indirekt die Vertreibung der lower class aus der Innenstadt, auch ohne daß erklärte Sanierungsprogramme nötig wären. Zur Senkung der Baukosten wird die Bohn, soweit möglich, durch die Vierfel mit der schlechtesten Bausubstanz gelegt, wo Grundstuckspreise und Entschädigungskosten gering sind und in denen "naturgemäß" die Gruppen mit dem niedrigsten Einkommen leben. (20) Damit kommi der bekonnte Mechanismus in Gang: Im gleichen Augenblick, da sich die Kommune durch den Bahnbau in verstärktem Maße verschulder und deshalts die Grundsteuer zur Aufbringung der Kosten erhoht, steigen die Grundstückspreise entlang der Trasse, vor allem aber im Umkreis der Bahnhoffe, weil die Nachfrage nach diesen durch den Bahnbau gut erschlossenen Grundstük = ken steigt. Diese erhöhten Bodenpreise werden der neuen Grundsteuereinschärzung zugrundege legt. Dies schlägt sich, ebenso wie der erhöhte "Wert" des Bodens, (der auf der Erwartung höherer Bodenrente beruht), in erhöhten Mieten nieder. Die Auswirkungen auf die Arbeiterfamilien mit geringem Einkommen, die jetzt dort wohnen, sind abzusehen: Während schon die Trassenführung selbst billige Wohnungen vernichtet, steigen die Mieten der Wohnungen an der Trasse unverhälmismäs w sig stork. Die bisherigen Mieter werden zum Auszug gezwungen; fur neue Mierer mit hoherem Einkommen ist die Bausubstanz zu schlecht. Die ollen Eigentümer mussen verkaufen, da sie für Abriß und Neubau ihrer Geböude zu finanzs chwach sind. Die Häuser gehen an große Baugesellschaften uber, die sie abreißen und das ganze Gebiet für hothere Einkommensklassen neu aufbauen.

Die dritte technokratische Planungsinitiative stoßt in einen Bereich, in dem sich, wie die Erfahrungen zeigen, der Widerstand der Betroffenen am ehesten herausbil den kann: die Stadtemeuerung. Die ersten Erfahrungen mit der Stadrerneuerung in San Frencisco zeigten schon deutlich, zu wessen Gunsten die von Konzerngruppen unterstutzten Planungen durchgefuhrt wurden, und sind daher fur uriser Theme von besonderem Interesse. Beim Golden Gateway, einem an der Küste gelegenen Projek? fur uberdurchschnitrlich hohe Einkommensklassen, des einen citen Rohprodukrenmarkt erseizte, ebenso wie beim Diamond Heights Projekt wurden selbst cuf unbeboutem Gelände nur Luxuswohnungen errichtet. Im ersten Sanierungsgebiet von Fillmore, wo fruher 6000 Schwarze lebten, vermochten nach der Enneuerung, bei der eine Reihe von teuren Wohnhäusern gebaut wurden, nur drei Familien in das 
Gebiet zuruckzuziehen. Einige schwarze Familien der Mittelschicht qgus anderen Gebieten zogen ein, aber nicht die Masse der früheren Ghertobewohner. 1964 begann die Sanierungsbehörde, in benachbarte Gebiete des Fillmore-Ghettas einzudringen. Mehr als 40000 Menschen, von denen $70 \%$ Schwarze sind, wurw den und werden von diesem Vorstoß betroffen. Als Hauptursache fü die Ausdehnung der Fillmore-Sanierung wurde die Sicherung der hohen Bodenwerte ongegeben, die durch die Aufwertung des ersten Sanierungsgebieies durch teure Wohnhäuser erreicht worden war. Den Plänen der Administration zurolge, kơnnen nur isolierte Ghetros wie Hunters Point, die weit von der Innenstedr entfernt sind, ohne die Vertreibung der gegenwärtigen Bevölkerung saniert werden. Im Stadtkern bleibt Sanierung identisch mit Evakuierung.

Das Regulativ dieser Praxis ist Produktion für den Markt zahlungskrafftiger Nachfrage. Der Leiter der Sanierungsbehörde von San Francisco, Justin Herman, drückte das so aus: "Jeder, der etwas nachdenkt und guten Willens ist, erkennt, daßes nicht Diskriminierung allein ist, sondern daß auch ein Mangel auf Seiten der Schwarzen besteht. Das moralische Ziel ist zwar - die Chancen cuszugleichen. Aber selbst wenn es theoretisch möglich wäre, Diskriminierung auszuschließen: Neger verdienen dennoch nicht genug Geld, um in San Francisco zu leben". (21) Dies ist die klare und bestimmte Aussage eines Sanierungspraktikers. Sie unterscheidet scharf zwischen moralischer Diskriminierung, die eventuell mit gutem Willen zu beseitigen ist, und "objektiver Unfähigkeit" "nämlich der beschrönkien Zahlungsfähigkeit der Lohnarbeirer im Kapitalsystem (von denen die Schwarzen freilich die am wenigsten zahlungsfähigen sind).

Dieser klaren Feststellung steht die beschönigende Funkrion von "Bürgerberatungs gruppen" wie der SAN FRANCISCO PLANING AND URBAN ASSOCIATION (SPUR) gegenuber. In einem Grußwort an eine Tagung von SPUR benannte ein Professor der University of California das strategische Dilemma der Sanierungsaktivität in San Francisco in sonntäglicher Reflexion: "San Francisco wird immer mehr polarisiert. Es besteht das unleugbare Bedurfnis, Leute der Mittelklasse mit Kindern herbeizuholen. Andererseits tragen wir auch die Verentwortung gegen = uber den Negern". (22) "Verantwortung" tragen die "Burgerberater" von SPUR aber vor allem gegenuber den Konzernen "die diese Gruppe zur Unterstützung der von ihnen vorangetriebenen Sanierung ins Leben gerufen haben und finanziell am Leben erholten. Im BLYTH-ZELLER BACH-COMMITTEE, dem Initiator der Burgerberatungsgruppe SPUR, sind U.a. vertreten: die Bank of America, Bechrel Corporation (die größre Baugesellschaft der Welt), Pacific Telephone, Pacific Gas and Electric, Standard Oil, Levi Strauss, American Trust Co., Magnins (großer Detailhandel der City) und Matson-Navigation. Die "Burger" "richtiger: die Bourgeois des BLYTH-ZELLERBACH-COMMITTEE kamen drei Jahre in voller Hohe für die Ausgaben von SPUR cuf und besorgen heure $50 \%$ ihrer Gelder; Verbindungsmonn zwischen den beiden Guppen wor der Finonzexperte 
Jerd Sullivan, der jahrelang sowohl als Vorsitzender von SPUR, wie auch des BLYTH-ZELLERBACH-COMMITTEE fungierte. John Hirten, ehemals leitender Direktor von SPUR, sorgte für die Verbindung zum BAY AREA COUNCIL, der 1945 gegründeteten Manger-Planungsgruppe, zu deren Verwaltung er gehörte. Gelegntlich gab einer der Direktoren der "Bürgerberatungsgruppe" SPUR seine spezifische "Verantwortung" gegenüber den Nicht-Eigentümern und Unterpriviligierten zu erkennen, indem er ihnen einfach die Qualifikation absprach, bei der Entscheidung über das Geschick ihres Gebietes eine Rolle zu spielen.

Entsprechend beurteilte denn auch die "Bürgerberater" der SAN FRANCISCO PLANING AND URBAN RENEWAL ASSOCIATION die Entstehung einer lokalen Basisorganisation, die sich im Herbst 1966 im Distrikt MISSION, einem innerstädtischen Ghetto, gebildet hatte. Für eine Sanierung des Distrikts MISSION hatte die Sanierungsbehörde von San Francisco einen Planungszuschuß des Bundes in Höhe von zwei Millionen Dollar beantragt. Der Plan der Stadtverwaltung wäre auf Sanierung des MISSION-Distriktes durch Vertreibung seiner gegenwärtigen Bevölkerung hinausgelaufen. Man wollte aus dem Viertel ein Wohngebiet für Beamte und Angestellte machen. Es war das Ziel des MISSION COUNCIL ON REDEVEL OPMENT, die Ausführung dieses Plans zu verhindern. Laut SPUR würde damit ein gefährlicher Präzedenzfall geschaffen dafür, "daß lautstarke und dro=" hende Gruppen - gleichgültig, ob sie verantwortungsvolle oder unverantworliche Positionen einnehmen - Maßnahmen blockieren könnten, die zur Aufrechterhaltung von Gesundheit und Wohlergehen der gesamten Gemeinde notwendig sind" -wenn der MISSION COUNCIL ON REDEVELOPMENT (MCOR) seinen Stand punkt in Bezug auf das Sanierungsprogramm für den Distrikt MISSION durchsetzen wïrde. (23)

Da hier der Sanierungskonflikt tatsächlich zur Mobilisierung der Massen führte und die organisatorische Konsolidierung gelang, erscheint das Beispiel des MISSION COUNCIL ON REDEVELOPMENT wichtig für die Beurteilung der Erfolgschancen und der politischen Perspektiven von lokaler Basisorganisation. Die spontane Entstehung und der tatsächliche organisatorische Erfolg des MCOR lassen dies Beispiel besonders geeignet erscheinen, um die Offesche These zu überprüfen, daß die Wahrnehmung von Handlungschancen im außerbetrieblichen Bereich "mit erfreulicher Folgerichtigkeit" zu einer Infragestellung der beiden "Systemprämissen politischer Reprä. sentation und privaiwirtschaftlicher Investitionssentscheidung" führe.

Der MISSION COUNCIL ON REDEVELOPMENT (MCOR) konstituierte sich als eine Koalition: als der Versuch einer organisatorischen Zusammenfassung aller sozialen, kulturellen und Bürgergruppen des Gebiets. Er unfaßte Leute aus allen möglichen "Wăhlergruppen": Schwarze, Braune, alteingesessene ethnische Gruppen, Jugendliche und Rentner, Eigenheimbesitzer und Mieter, Arbeiter und kleine Geschäfsleute. Der MCOR versuchte, Solidarität über die ldentifikation mit dem Gebiet, dem "turf", herzustellen: Was Haben " $d i e$ " mit unserem Viertel zu fun? Wenn 
man von einer Ideologie des MCOR sprechen kann, so war ihr Kernsatz: Laßt die Leute selber entscheiden! - also die Forderung nach Gemeinde-Selbstbestimmung.

1968 reorganisierte sich der MCOR als umfassende Vereinigung von Delegierten aus 75 lokalen Gruppen und anderte seinen Namen in MISSION COALITION OR GANIZATION (MCO). Vorangegangen war die Reorganisaton von Stadt und Gemeinde in Planungsfragen: Die Bundesregierung hatte das Musterstädte-Programm aufgestellt, um damit alle Bundesmittel für die städtisçhen Dienstleistungen und für die Stadterneverung einheitlich verwalten zu können. Die Stadt San Francisco gab die Absicht bekannt, einen Planungszuschuß zur Untersuchung der Möglichkeiten von Gebietsrenovierung und Sanierung in Mission und in Hunters Point zu beantragen. Schon Anfang 1967 hatte der MCOR gewaltigen Widerstand gegen die Vorstellung einer Sanierung ohne Kontrolle durch die Betroffenen entfaltet. Die MISSION COALTION ORGANIZATION setzte das politische Programm des MCOR verschärff fort. Sie forderte Vetorecht für das Stadtviertel bei allen fälligen Entscheidungen, wenn überhaupt saniert werden sollte. Alle Planungsvorschläge müßten zuerst von der COALITION gebilligt werden, bevor sie der Stadtverwaltung vorgelegt würden. Die MISSION COALITION ORGANIZATION verlangte, daß zwei Drittel aller Mitglieder der CITY DEMONSTRATION AGENCY (CDA), des Ausschusses, der das Programm in Mission durchführen sollte, Bewohner des Gebietes sein müßten. Sie sollten von der Koalition gewählt und von ihr abrufbar sein. Nach Erfüllung dieser ersten Forderung sollte sich der Ausschuß mit der MISSION COALITION in Verbindung setzen, um die sozialen Bedürfnisse zu ermitteln, Prioritäten zu setzen und die Ziele des Programms zu bestimmen. Bis zum Frühiahr 1969 hatte die MCO ihre Basis, im Mission-Distrikt wohnende Spanier und Mexikaner, derart aktiviert, daß sie den Bürgermeister von San Francisco zu einem Abkommen zwingen konnten: Die Planungsbefugnisse zur Verwendung der Bundesmittel aus dem Musterstädte-Programm sollten ihr zugesprochen werden.

Ist Gemeindekontrolle über die Bundesmittel aus dem Modellstädte-Programm, also Mitverfügung über Mittel aus dem offentlichen Haushalt, ein Sieg für die bislang Unterpriviligierten? In einem strategischen Kalkül, das von der Interessenstruktur und dem Machtgefälle im Rahmen der Gesamtgesellschaft ausgeht, beantworten die Autoren der Fallstudie von San Francisco, Beagle, Haber und Wellman, diese Frage.

"Einerseits ist die Antwort ein deutliches Ja. Der Kampf um Gemeindekontrolle ist wichtig. Gegen die Mißachtung der Volkssouveränität muß Widerstand geleistef werden. Die technokratische Koordination von Geschäftswelt und Exekutive muß verhindert werden. Und zumindest kurzfristig können sich viele wesent= liche Vorteile daraus ergeben. Es sollte jedoch klar sein, daß Gemeindekontrolle allein keine Lösung für die Nachbarschaften von Mission sein kann. Sie hat im besten Fall eine ou $\mathrm{f} \mathrm{ch}$ ie bende Wirkung gegen den Gesamtplan der 
der Konzerne, der für Mission die Auslöschung bedeutet. Die letzllich bestimmenden Kräfte, die die Zukunft des Ge bietes formen, entziehen sich dem Einfluß der Model $\mid$ st ad te $p$ la $n$ er. Der alte Sanierungs-Evakuierungs-Plan für Mission ist nicht aufgegeben worden. Die allgemeinere Strategie der Konzernplaner bestimmt die Bedingungen, innerhalb derer die Bewohner handeln müssen". (24)

Zur Begründung dieser These bringen Becgle, Haber und Wellman folgendes vor: Um das Schnellverkehrssystem und die Stadterneverung zu finanzieren und zweife $\mathrm{l}=$ los auch die Kosten der regionalen Verwaltung, werden sich die Konzernplaner weiterhin auf dieselbe Steuergrundlage verlassen "die für alle städtischen Funktionen angezapft worden ist : die Vermögensstever. Nach der steverlichen Einschärtzung der Vermögensobjekte wird die Höhe der Besteuerung entsprechend dem Finanzbedarf der Kommune festgesetzt. Die steigenden Grundstückspreise an der Schnellverkehrslinie mitten in Mission und in der Nähe anderer Neubaugebiete finden entsprechend der "Steuergerechtigkeit" ihren Ausdruck in einer höheren steverlichen Veranlagung der Objekte. Die Folgen sind absehbar:

"Die erhöten Stevern werden die Mieten in den bereits herabgewirtschafteten Wohnungen für Leute mit geringem Einkommen in die Höhe treiben und die kleinen, nachbarschaftsorientierten Geschäfte noch weniger profitabel machen. Der Markt wird weiterhin das Seine tun, und zwar unerbittlich: Profitorientierte Baufirmen werden die bereits ausgepowerten Eigentümer aufkaufen und neue Gebäude errichten. Neue Geschäfte werden für die neuen, wohlhabenderen Leute entstehen, die sie profitabel machen können. Dieser Vorgang mag nicht so sauber und schnell vor sich gehen wie bei der Flachensanierung, wo die Planierraupe in einem grausamen Schub die Nachbarschaft zerst art. Das Ergebnis aber wird dasselbe sein: das schrittweise Verschwinden des Viertels, wobei Baufirmen die Kerngebiete entlang der Schnellbahnlinie übernehmen, dann ihre Eingriffe erweitern und festigen, indem sie die noch verbliebenen Bewohner erst einmal ausgrenzen mit Parks, neven Schulen oder anderem, barriereartig dazwischengeschobenen Einrichtungen, die geplant werden, um die "allgemeine Wohlfahrt" zu fördern. Die Kehrseite der Medaille ist jedoch ein Prozeß der blo ckwe is e n Zerstörung des Viertels. . und dies ist ein Prozeß, den umzukehren die MISSION COALITION und ähnliche, auf Gemeindekontrolle abzielende Organisationen nicht in der Lage sein werden. Die Kräfte, die diesen Prozeß erzwingen, kommen von außen auf die Gemeinde zu: die Finanzierung der städtischen Ausgaben über Vermögenssteuern, die Festsetzung von Grundstückswerten und von Steuersätzen" . (25)

Beagle, Haber und Wellman stellen abschließend fest: Auch wenn die MISSION COALITION ORGANIZATION einen vorzüglichen, ihren Bedürfnissen entsprechenden Alternativplan entwickle, werde es ihr nicht gelingen, ihn in die Praxis umzusetzen. Die Situation sei áhnlich wie bei der Gemeindekontrolle über die Schulen, wenn die Bewohner keine Kontrolle über die Finanzierung der Erziehung und uber die Rekrutierung und Ausbildung der Lehrer haben; oder wie die Ge- 
meindekontrolle über die Polizei, wenn die Geseize und das Gerichtsverfahren unverändert bleiben und wenn der Strafvollzug mechanisiert, mit Computern rationalisiert und regional organisiert werde; oder Gemeindekontrolle über die Fürsorgeverwaltung, wenn die Höhe der Steuermittel und die Richtlinien vom Staat oder den Bundesbehörden festgesetzt werden.

"In all diesen Fällen kann, wie im Fall der Sanierung, die Gemeindekontrolle gedankenlosen und willkürlichen Mißbrauch ausschließen. Die irrationale und überflüssige Ausbeutung kann verhindert werden, wobei Community Power, Geldschneider, Prügelpolizisten, habgierige Slumbarone, demuitigende Fürsorger und dergleichen in Schach halten kann. Aber das Ziel, das Unvermeidliche zu humanisieren, ist vollauf mit den Konzerninteressen vereinbar. Es ist schon längst eine der Grundlagen ihres Herangehens an die "städtische Krise". G em e in d e kontrolle leistet die Anpassung an das Unvermeidliche und Unabänderliche mit wenigerErbitterung und Ungerechtigkeit. Aber das Recht, die Liquim dation der eigenen Nachbarschaft anzuführen, ist kein Triumph der Demokratie。 (26)

Als entscheidende Einsicht ergibt sich aus dieser Analyse, daß politische Macht uber ein Gebiet nicht zu erreichen ist mit Gemeindeselbstbestimmung. Denn der Bereich, auf den sich Gemeinde-Selbstkontrolle erstrecken kann, ist viel zu begrenzt.

Doch bei dieser Einsicht bleiben Beagle, Haber und Wellman auch stehen. Sie rem duzieren damit das Problem auf eines der Reichweite der Kontrolle. Die politische Alternative zur Organisierung lokaler Situationsgruppen wäre damit die Organisierung nationaler Situationsgruppen, die das den Kommunen übergeordnete politische Zentrum zum Adressaten ih rer Forderungen machen. Die Kritik der Organisationsform des Widerstandes beschränkt sich auf eine Kritik ihres lokalen Charakters. Mit einer solchen Kritik können zwar immerhin lllusionen der Art, wie Offe sie den Stadtplanern vortrüumt, zerstört werden, ihr ideologischer Charakter kann aufgezeigt werden, doch trifft diese Kritik nicht den Angelpunkt des Problems: die Herrschaft des Kapitals und die Unterwerfung aller Lebensbereiche der arbeitenden oder auch arbeitslosen Menschen unter die Bedürfnisse seiner Verwertung. Das Problem bleibt für sie eines der Begrenztheit der Gemeindekontrolle gegenüber Mächten, die sich auf einer Ebene formieren, die der Gemeinde übergeordnet ist. Zwar ist ihnen bewußt, daß zu diesen übergeordneten Mächten nicht allein der Staat, sondern auch die Konzerne gehören: "Es ist unbedingt notwendig", sagen sie " "iene Aspekte der politischen Ökonomie zu bestimmen die das konkrete Programm beeinflussen, zu dessen Durchsetzung Gemeindekontrolle nur als Instrument benutzt wird. Die Analyse der Konzernstrategie nennt einige Bedingungen, die die Reichweite der Programme beschrönken: sie verweisen auf Gebiete, wo mehr als "turf power" erforderlich ist". (27) Sie sehen auch, daß die staatliche Steuergesetzgebung die Profite der Konzerne schützt. Doch 
wenn die Autoren dann als politisch-strategische Konsequenz ihrer Analyse for dern, die Profite mithilfe sozialreformerischer Stevergesetzgebung zu sozialisieren, so wird deutlich, daß sie die Macht der Konzerne nur als politische Machi begreifen, der mit politischen Mittein, nåmlich einer sozialreformerisch orientierten Staatsgewalt, entgegentreten werden könne. Demgemäß können sich auch nichr die richtige strategisch-politische Konsequenz aus ihrer Analyse des Scheiterns von Community Power ziehen: die Notwendigkeit der gemeinsamen Organisation der Lohnarbeiter und der industriellen Reservearmee als Klasse, die dem Kapital nicht nur als objektiv von ihm erzeugrer, sondern auch subjektiv seiner Macht bewuBter Widerspruch entgegentritt.

Doch fassen wir noch einmal die Ergebnisse der San-Francisco-Srudie zusammen, um sie den anfangs vorgestellten Offeschen Thesen gegenuberzustellen. Die Autoren der San-Francisco- Studie stellen an ihrem Beispiel mit aller Klarheit die begrenzte politische Reichweite lokaler Selbstorganisation dar. Lokale Situationsgruppen können zwar gegen den politisch zusammengefaßten Ausdruck der naturwỉchsigen Tendenzen der Kapitalverwertung, gegen die Sanierungsbe= hörden, sich zur Wehr zu setzen versuchen. Sie können diese Tendenzen selbst aber nicht treffen und in ihrer Richtung und Wirkungsweise umkehren. Erinnern wir uns an die Theorie von Offe, wie er sie vor den Stadtplanern ausgebreitet hat: Sachzwang herrsche dort, wo die Institutionen der Öffentlichkeit vertrocknet seien. Es bedürfe also der Mabilisierung der Betroffenen, ihrer Fahigkeit, ihre Interessen und Bedürfnisse zu formulieren, donn sei in den Bereichen, die in einer "relativen Ferne" von den zentralen Bereichen Wirtschaft, Militär, Politik ständen und in denen demgemäß ein gewisser Spielraum zur Veränderung gegeben sei, ein Zerbrechen der "Sachzwånge" möglich.

Demgegenüber zeigt der beschriebene Versuch einer Mobilisierung der Öffentlichkeit zur Durchbrechung von "Sachzwängen" in San Francisco genau das Gegenteil: Zum einen stellen sich die "Sachzwänge" ganz eindeutig als solche der Kapitalverwertung heraus. Stadtsanierung erweist sich als keineswegs "relativ fern" von dem zentralen Bereich "der Wirtschaft", sondern als eindeutig strukturiert durch besondere Interessen großer Kapitale. Diese schaffen sich innerhalb einer Region ein Zenirum, an dem sie ihre Zirkulation, Kouf und Verkauf von Waren, die damit verbundenen Geld-und Kreditgeschäfte, Versicherungen und Verwaltung möglichst effektiv betreiben können. Solche rầumliche Zentrierung der Zirkulation des Kapitals wirkt sich auf der einen Seite als Erhöhung der Umschlagsgeschwindigkeit des Kapitals, auf der anderen Seite als Verminderung der foux frais, der roten Kosten der Zirkulation des Kopitals aus. Beide Momente wirken sich als Erhöhung der Profitrate der einzelnen Kapitalisten aus. Zudem ermöglicht die Ausrichtung einer ganzen Region auf ein Zentrum, in dem der Uma schlag der großen Kapitale stattfindet, dem Großkapital, noch verbliebene Reste mittleren Industrie-, Handels- oder Bankkapitals niederzukonkurrieren oder zur Fusion zu zwingen. Das Kapital hat also die Tendenz, Metropolen seines Um- 
schlogs zu schaffen, ohne Rucksicht auf die damit entstehenden Probleme, ja Karastrophen städtischer Infrastruktur. Dies zeigt sich nicht nur an dem Beispi el San Francisco, sondern in gleicher wünschenswerter Klarheit z. B. an den Städten München und Frankfurt in der BRD.(28)

Die auf der Grundlage solcher Tendenzen des Kapitals entstehenden Planungskommissionen in San Francisco litten nicht etwa daran, daß sie auf eine "vertrocknete Offentlichkei $t^{31}$ trafen - im Gegenteil: Das COMMITTEE FOR ECONOMIC DEVELOPMENT, welches sich zum Zweck der regionalen Planung zusammengefunden hatte, war als Vereinigung der größten Konzerne systematisch bestrebt, die leizten Reste einer selbständig fungierenden Öffentlichkeit, die verstreuten Kommunalverwaltungen zu zerschlagen, um an ihre Stelle eine zentrale Verwalfung mir zentrolen Planungsmöglichkeiten zu setzen, welche in ihrer Effizienz nicht den Störungen widerspenstiger Kleinkommunen ausgeseizł isł. Das für die Betroffenen Verhångnisvolle an der Stad tsanierung lag also nicht etwa am Fehlen einer zentralen Planungsinstitution, wie Offe meint, sondern an der Unterwer= fung der Wohn = und Lebensbedürfnisse der Bevölkerung einer ganzen Region unter die in diesem Falle gemeinsamen Interessen großer Kapitale, organisiert eben in einer zentralen Planungsinstitution des Kapitals. Alle Versuche einer "wiederbel ebten Offentlichkeit" sich den Planungszielen zur "Stadtsanierung" des Großkapitals zu widersetzen, scheiterten, indem sie sich als "Situationsgruppe" formulierten. Eine bestimmte Wohnsituation bildete den gemeinsamen Interessenhintergrund der Organisierung. Doch der Widerspruch zum Kapital ist nich der wohnbedurfige Bürger, sondern der dem Kapital den Mehrwert zuschuftende Lohnarbeiter und die der Klasse der Lohnarbeiter objektiv zugehörige industrielle Reservaarmee. So kann auch eine Organisation der Wohnbedürftigen zu einer ihre Interessen formulierenden "Situationsgruppe" den großen Kapitalen ernsthaft keinen Widerstand entgegensetzen, werden diese ja nicht einmal als Adressat der Forderungen und wirklicher Gegner begriffen. Gercde dies ist aber auch die Grundlage der Möglichkeit, solcherart auf eine "Situation" -hier die Wohnsituation- beschränkte politische Mobilisierungsversuche zu integrieren in die Planungsstrategie der Kanzerne selbst: "Gemeindekontrolle leistet die Anpassung an das Unvermeidliche und Unabänderliche mit weniger Erbitterung"

Der Adressat einer solchen "Situationsgruppe" ist al lenfalls die Stadtverwaltung, und nicht sie ist es, die die Interessen des Kapitals definiert, sondern genou um. gekehrt: Die offentlichen Institutionen sind in ihren Infrastrukturplanungen selbst bestimmt durch die Verwertungszwänge des Kapitals, finde dies nun seinen Ausdruck in einer Personalunion zwischen politischer Macht und Kapital oder nicht. Der Spielraum, der der Stadtverwoltung gegenüber den Interessen der großen Kopitale gegeben ist, erweist sich als äußerst gering. Denn der Erfolg, den die MISSION COALITION ORGANIZATION verbuchen konnte, indem sie die Planungsbefugnis über Bundesmittel zur Stadtsanierung erhielt, bricht sich an dem Prozeß der Verteuerung der Grundstückspreise im Zuge der Strukturierung der 
der Stadt nach den kapitalistischen Notwendigkeiten. Was die Stadtverwaltung mit der einen Hand gab, nämlich die Verfügung über beschränkte Mittel zur Sanierung, das machre sie mit der anderen Hand unwirksam, indem sie die gestiegene Grundstücksbewertung als Grundlage erhöhter Steuern benutzte und auf diese Weise an der Zerschlagung der Möglichkeit einer Sanierung des Distrikts Mission im Interesse der dort wohnenden Schichten der Arbeiterklasse mitwirkte. Auch hier wieder handelt die Stadtverwaltung nicht eigenständig und im Rahmen eines ihr gegebenen Spielraums, sondern folgt ganz einfoch in ihrer Besteuerung dem Prozeß der Verteuerung von Grund und Boden im Gefolge der "Entstehung zahlungskräftiger Nachfrage nach knappen Mitreln", wie die bürgerliche Ö́konomie hier sagen würde. Die Grundlage der Entstehung dieser "zahlungskräftigen Nachfrage" ist hinwiederum die Umformung der Stadt zum Zentrum der Zirkularion der großen Einzelkapitale. Auf dieser Grundlage bewegt sich dann auch die zweite Kapitalgruppe, die hier als Handelnder auftritt und an der sich der Widerstand der wohnbedürfigen Bürger bricht: die großen Baufirmen und die Grundstücksund Wohnungsmakler, denen neben den Wohnungen der Arbeiter auch der kleine städtische Grundstücks- und Hausbesitz zum Opfer fällt. Sie werden van den lokalen, auf die Stadtverwaltung hin gerichteten Widerstand der "Situationsgruppe" ebensowenig getroffen wie die großen Kapitale, die die Sanierung in Gong setzten.

\section{DIE VERWEIGERUNG DER ARBEITER GEGENŮBER "AUSSERBETRIEBLICHER MITBESTIMMUNG"}

Die Klassenlaye des Proletariats in den USA ist hauptsächlich darin von der Situation in der BRD verschieden, daß wegen der Existenz einer ausgedehnten Reservearmee in den USA zum einen der betriiebliche Kampf erschwert ist und zum andern der Kampf im Stadtteil zum besonderen Schauplatz der Klassenauseinandersetzungen wurde. In der BRD erleben dagegen auch die Teile des Proletariats im Betrieb das Zentrum der Ausbeutung, die von der Aktion des konzentrierten und in staatlichen Agenturen politisch zusammengefaßten Kapitals in der Reproduktionssphäre am stärksten betroffen sind. Die Weígerung der Arbeiter, im Stadtteil den Schauplatz ihrer Kämpfe zu sehen, ihre ablehnende Antwort auf Mobilisierungsversuche "professionalisierier Intelligenz" soll im folgenden dar gestellt werden.

Das Sanierungsgebiet Kreuzberg umfasst ein dicht bebautes 107 ha großes Gebiet am Rande des Berliner City-Bereichs. Es ist außerordentlich gut mit Massenverkehrsmitteln (2 U-Bahnlinien mit $4 \mathrm{U}$-Bahnstationen, mehrere Buslinien) an die City angeschlossen und besitzt ebenso gute Verbindungen zu den übrigen randşrädtischen Gebieten. 1961 lebten hier 37000 Menschen, nach Angaben der 
Wbhnungszåhlung von 1961 waren $70 \%$ der Erwerbståtigen Arbeiter. Die Gewerbestruktur ist gekennzeichner durch ein Vielzahl kleiner und kleinster Betriebe: Bei der Gewerbezóhlung 1961 wurden fast 3000 Betriebe ermittelt mi insgesamt etwa 20000 Beschäftigten. Diese Betriebe stellen meist Produkte her, deren Fertigung sich für die Großbetriebe, von denen sie teilweise direkt als Zulieferer abhängig sind, nicht (oder noch nicht) lohnt. Einige dieser Kleinbetriebe konkurrieren auch mił größeren, indem sie schneller liefern als diese und mit geringeren Profiten vorlieb nehmen. Der Handel ist in einer großen Zahl von Kleinstläden verteilt uber das gesamte Gebiet und verdichtet sich an zwei sich kreuzenden Schwerachsen.

Die Bausubstanz ist fast durchweg 70 - 120 Jahre alt, ohne daß kontinuierlich größere Erneuserungsarbeiten vorgenommen worden wären. Mit der Erklärung des Viertels zum Sanierungsgebiet 1963 wurde in dem Gebiet der Verfall der Bausubstanz beschleunigt und von seiten der Verwaltung mit der Schließung von offentlichen Einrichtungen begonnen: Im Sommer 1970 wurde das Krankenhaus Bethanien geschlossen, obwohl es, wie Experten vorgeschlagen hatten, sinnvollerweise mit speziellen Funktionen weitergeführt worden wäre. Der Neubau des Krankenhauses Am Urban außerhalb des Gebiets kann nur teilsweisen Ersatz leisten. Ebenso wird vom Berliner Senat mit Schließung und Nicht-Erweiterung von Kindergårten, Schulen und Poståmtern die Strategie verfolgt, das Gebiet im Hinblick auf Sozialeinrichtungen und Dienstleistungen auszutrocknen. Parallel geht die systematische Herabwirtschaftung der Wohngebäude, die fast durchweg nach dem Krieg nicht renoviert worden sind. Beides soll die Notwendigkeit der Flächensanierung des gesamten Gebiets augenscheinlich machen und ihre Vorbedingung, die Evakuierung der Arbeiterbevölkerung, als einzige Möglichkeił erscheinen lassen. Die neuen Wohnungen nach der Flächensanierung werden aufgrund der gegebenen Bedingungen auf dem Wohnungsmarkf für die überwiegende Mehrzahl der jetztigen Bewohner unerschwinglich sein.

Die Analyse der Sanierungsplanung für Kreuzberg zeigt die enge Verflechtung der Stadtsanierung mit den Interessen des großen Kapitals auch in der BRD, speziell in Westberlin, die sich weder in ihren grundlegenden Strukturen noch in ihren Erscheinungsformen prinzipiell von den amerikanischen Vorbildern unterscheidet:

Die Konzeption der Neubebauung, wie sie vom Berliner Senat nach und nach vorgelegt wurde, ist strukturiert durch eine Verkehrsplanung, die die bisher fehlende Anbindung des Gebiets an das innerstädtische Autobahnnetz leistet: Zwei Tangenten des Autobahnnetzes werden sich mitten im Gebiet schneiden und es in vier getrennte Teile zerlegen. Die dann auch für den Autoverkehr außerordent= lich günstige Verkehrssituation wird das Gebiet noch enger mit den zentralen City-Bereichen wie mit den entlegeneren Teilen der Stadt und damit mit deren Warenmärkten und Arbeitsplätzen sowie mit deren Kaufkraftpotential verbinden. 
In unmittelbarer Nähe der Kreuzungen der Autobahnen und U-Bahnlinien - im Schwerpunkt des Einzugsbereichs der potentiellen Konsumenten - wird ein zentraler Marktbereich geschaffen, der die Konzentration und Rationalisierung des Warenumschlas gewährleisten wird. (29) Daß an diesem Knotenpunkt bereits lange vor dem Kahlschlag des ganzen Viertels und der endgültigen Durchführung der Pla nungen die Bodenpreise bereits phantastisch gestiegen sind, bedarf wohl kaum noch der Erwähnung (30) . Im übrigen basiert die Flächennutzungsplanung des Gebietes auf klarer Trennung von Wohngebieten mit möglichst hohem "Wohnwert" und einigen Gewerbeblocks an den Autobahnen (wo sich wegen des Verkehrslärms Wohnungen schlechter vermieten lassen). Hier werden unter Verwendung gut erhaltener alter Gewerbegebåude die produktivsten Betriebe möglichst belassen, wo sie sind oder auch hierher umgesetzt.

Erstes Ziel der Sanierung in Kreuzberg, so könnte man zusammenfassen, isł̂ es, Schneisen für das Berliner Autobahnsystem zu schlagen und wertvollen innerstädtischen Boden profitablerer Nutzung zuzuführen. Die Neuordnung übergeht die Bedürnisse der jetzigen Bewohner und schert sich auch wenig um die Interessen der Masse der Kleinkapitalisten. "Nur durch rechtzeitig eingeleitete Erneuerungsmaßnahmen kann verhindert werden, daß wichtige und wertvolle Innenstadt gebiete die ihrer Lage entsprechende Bedeutung als Wohn- und Arbeitsgebiete verlieren", hat der Senat selbst recht klar bereits im ersten Sanierungsbericht 1963 formuliert. (31)

Die Grundprinzipien der Flächennutzungsplanung von Agglomerationstäumen unter scheiden sich wenig in unseren beiden Beispielen San Francisco und Berlin. In beiden Fällen erzeugen ähnliche ökonomische Ursachen bei ähnlichem Entwicklungso grad der Transportmittel (was natürlich nicht unabhängig voneinander ist) ähnliche Flächennutzungsschemata der kapitalistischen Großstadt .

Ein von den USA abweichendes Moment der Planungsziele resultiert daraus, daß in der BRD - im Gegensatz zU den USA - die Arbeitskraft relativ knapp ist. Fast 20000 Lohnabhångige sind im Sanierungsgebiet Kreuzberg in kleineren und mittleren Produktionsståtten beschäftigt. Diese Betriebe werfen jetzt noch Profite ab, weil die Gewerberaummieten extrem niedrig sind und die Arbeitskräfte relativ billig. Denn die Arbeiter wohnen zum großen Teil in der Nähe in billigen Wohnungen und sind deshalb vielfach bereit, um niedrigeren Lohn zu arbeiten, da der höhere Lohn in einem moderneren, aber weit entfernten am Stadtrand gelegenen Betrieb wieder einen Abzug durch lange Wegezeiten und Fahrtkosten erfährt. Mit der Sanierung wird diesen Kleinbetrieben die letzte Grundlage ihrer ohnehin abnehmenden Konkurrenzfähigkeit entzogen. Daß die Großbetriebe damit ein zusätzliches Potential an qualifizierten Arbeitskräften in ihren Verwertungsprozeß integrieren können, stellt ein wesentliches Element i h $r \in s$ Interesses an der Sanierung dar. Entsprechend warten die großen Kaufhäuser auf die Einverleibung jener Arbeitskräfte, die jetzł noch in den zahlreichen kleinen Geschäften des 
Gebietes gebunden sind. Nach der "Unsetzung" wird des Budgef der Arbeirerfomilie durch die Miete der neven Wohnung so belastet, daß jetz" auch die Frau mitarbeiten muß. Die Industrie erhält als Zugabe der Sanierung, was sie seit der vollen Ausschöpfung der Aibeitskraft ressourcen in Berlin permanent verlangte: die billige Arbeitskraft der Frau.

Es wird deutlich, daß Sanierung nicht irgend ein "Gesundmachen" eines Viertels bedeutet, das man durchführen oder lassen kann. Sanierung ist vielmehr als Modernisierung der materiellen Infrastruktur eine der essentiellen Bedingungen dep Verwertung der fortgeschrittenen Kapitale einer Region. Nicht die "vercltete Bausubstanz $z^{\text {in }}$ im Viertel selbst ist entscheidender Grund fur die Sanierung, sondern die inadäquate Bodennutzung des Sanierungsgebietes relativ zum gesamten Berliner Agglomerotionsraum. Die Notwendigkeit permanenter Anpassung der Infrastrukfur der Agglomerati onsräume an die Bedurfnisse der Kapitalverweriung gilt nicht nur fur die spezifische Situation von San Francisco oder Berlin. Vielmehr mußten in allen Agglomerationsräumen die jeweils mächtigsten Kapitale stets irgendwann die Anpassung der Infrastruktur an ihre Bedurfnisse erzwingen. (32) Unter den Bedingungen der freien Konkurrenz waren die infrastrukturellen Maßnahmen meist erst im Nachhinein gemacht worden ${ }_{8}$ wenn die Verwertung einer großen Zahl der betroffenen lokalen Kapitale ernsthaft bedroht war. Mit fortschreitender Kapitalkonzentration jedoch werden infrastrukturelle Investitionen schließlich zum Mittel, um y o r ab günstige Verwertungsmöglichkeiten für die Monopole zu schaffen. Dabei zwingt die Konkurrenz der Regionen, respektive der in ihnen lokalisierten Kapitale, diese nicht nur zum Kampf um möglichst große Anteile aus den staatlichen Fonds, sondern auch um möglichst effek tive Verwendung dieser Ressourcen entsprechend ihren jeweiligen Interessen. Daher ist der verbreitete Erklärungsversuch der Berliner Sanierungsvorhaben als direkte Subventionierung eines beschäftigungslosen überdimensionierten Produktzweiges, des Baugewerbes, völlig abwegig. Das Bau gewerbe selbst wäre zu wenig mächtig - besonders im gegenwärtigen Zustand seiner Rückständigkeit und Kleinheit der Einzelkapitale - um sich derartige Becchtung seines Profitinteresses zu sichern, zumal in einem Zeitraum, in dem alle anderen Unternehmer uber Mangel an Arbeitskröften klagen. So ist denn auch einer der wesentlichen Grunde für den langsamen Fortgang der Sanierung die Abneigung der Berliner Industrie gegen den Abriß von Wohnungen, in denen billige Arbeitskräfte untergebracht werden können. (33)

Der stringente Zusammenhang zwischen dem allgemeinen Inferesse des großen Kapitals an Rationalisierung des gesamten Wirtschaftsraumes des Strdtstaates Berlin und der Sanierung von Kreuzberg wurde von den Studenten, die den Widersiand der betroffenen Arbeiter organisieren wollten, nicht untersucht. Sie machten für die Kahlschlagsanierung hauptsöchlich die Verbindung der Berliner Bauindustrie mit einer unfähigen und korrupten Planungsinstanz verantwortlich. In Über- 
tragung ihrer besonderen Erfahrungen aus der Hochschulrebellion, ihren Erfolgen gegenuber einer antiquierten Universitätsbürokratie entwickelten sie quasi naturwüchsige Vorstellungen - wie sie Offe systematisiert hat - von "peripheren Bereichen" "in denen die Herstellung einer politisierten Öffentlichkeit bereits genüge, um den Staai zum Ruckzug zu zwingen. Denn dieser würde es nicht wagen können, offen seine sozialstaatliche und demokratische Maske fallen zu lassen. So richteren die Basisgruppe Sanierungsgebiet Kreuzberg ihr organisatorisches Konzept nach der Vorsłellung aus, daß unter dem Druck der Situationsgruppe der Sanierungsbetroffenen einerseits und dem Legitinationsanspruch des Staates andererseits die Kahlschlagsanierung verhindert werden könnte.

Ganz wie Offe als Funktion der "professionalisierten Intelligenz" konzipiert hatte, versuchte die Gruppe, die sich aus Architektur - und Soziologieshudenten zusammensetzte, mithilfe von Alternativplänen zu den offiziellen Sanierungsprogrammen den Druck der Betroffenen zu mobilisieren. In den Seminaren war efwo folgendermaßen argumentiert worden:

Beim Sanierungsgebiet Kreuzberg handele es sich um ein sozid homogenes Arbeiterviertel. Die vorhondenen Straßen, Plätze, Grüflachen und Kneipen, die Dichte der Bebauung begünstigten die Kommunikation zwischen den Bewohnern . Diese "Trivialkommunikation" werde sich politisch artikulieren, wenn durch die Agitation der Gruppe die drohende Zerstörung des Viertels und die Möglichkeit solidarischer Aktion aufgezeigt würden. Dies jedoch bedürfe der Darstellung der konkreten Utopie, die an "Grundriss, Aufriß und Vogelperspektive" vorzustellen eine Aufgabe der "revolutionären Berufspraxis" von Architekten und Soziologen sei.

Jedoch: Trotz intensiver Agitation unter den Bewohnern über die Folgen der Kahlschlagsanierung und die Möglichkeiten von besseren Alternativen; trotz der in einer Stufrenfolge aufgebauten Organisationsversuche von Demonstrationen über Protestversammlungen zu Mieterräten; trotz des Gebrauchs einer Vielzahl agitatori scher Mittel - regelmäßige Flugblätter, Agitationsgespräche in den Wohnungen, auf der Straße und in Kneipen über die Sanierung, Verkauf einer Sanierungszeitung, Straßentheater, regelmåßige juristische Mieterberatung; und obwohl die Gruppe in informellen Kontakten mit der Arbeiterbevölkerung weitgehende $\mathrm{Zu}$ stimmung erfuhr zu den Forderungen: "Stop der Kahlschlagsanierung, bis ausreichende und billige Neubouwohnungen erstellt sind!" - "Kampf dem Weißen Kreis" "Erhaliung des Bethenien-Krankenhauses!" - Die Solidarisierung der Betroffenen zur Organisation praktischen Widerstandes gegen die Sanierungsstrategie des Senats kam nicht zustande.

Diese Erfahrungen bestätigten sich auch in der gezielten Agitation eines råumungsbetroffenen Straßenzuges im Herbst 1969. Nicht einmal hier gelang es, das Konzept der Mieterselbstorganisation in Form einer kollektiven Auszugsverweigerung zu realisieren. Viele Bewohner hatten einen Besuch auf Senatsämtern hinter sich, 
wo sie nach Ersatzwohnungen zu akzeptablen Preisen gefragi hatten und mit un w zumutbaren Angeboten abgespeist worden waren. Die Individualisierung in der außerbetrieblichen Sphäre, in der keinerlei Arbeit eporganisation glaubwürdige Perspektiven solidarischen Handelns bietet, konnte nur ohnmächtigen Defetismus hinterlassen. Hier gibt es keine Erfahrung solidarischen Heandelns, die im Produktionsprozeß wenigstens durch die Organisation des Arbeitsprozesses selbsi angelegt ist. Daher bestand für die Räumungsbetroffenen individuell nur die $A l-$ ternative zwischen diskriminierender Fürsorge und individueller Selbsthilfe be stand. Daraus bot auch die Selbstorganisation der Mieter keinen realen Ausweg. Das trat in Gesprächen, in denen die Möglichkeit kollektiver Auszugsverweige rung durchkonstruiert wurde, deutlich zutage. Die Perspektive der Auszugsverweigerung mobilisierte nur die panische Angst vor dem Obdachlosenasyl.

Die Forderung, die Kahlschlagsanierung zu stoppen, bis ausreichende und billige Neubauwohnungen erstellt wirden, war zwar eine unmittelbar einsichtige Antwort auf den Räumungsbefehl. Die Arbeiter hatten jedoch die realistische Einschåtzung, daß die Errichtung billiger, unter dem Miespreis-Niveau des Sozialen Wohnungsbaus liegender Neubauwohnungen nicht durch eine lokale Gruppe erzwungen werden kann. Sie konnten aber - das war ihnen bewußt - auch nicht auf Unterstutzung durch die Gewerkschaften hoffen. Es bestand keinerlei Erwartung, daß diese im Sanierungsproblem aktiv würden. Die Rolle der gewerkschafteigenen "Neuen Heimar" bei Sanierungs- und Neubauprojekten war durchweg bekannt und wurde als Mitprofitieren der Gewerkschaften an überhohen Mieten illusionslos dargestellt.

Was immer die Einstellungen der agitierten Arbeiter zu den Gewerkschaften gewesen sein mochten: Im kritischen Bezug ouf das gewerkschafłseigene Baunnternehmen zeigte sich das Bewußtsein der Arbeiter, von opportunistischen Gewerkschaftsführern verraten zu sein. Hatten sie schon mit thresgleichen diese Erfahrung machen müssen, so mußte das Mißtrauen gegenüber "Anwälten" ihrer Interessen aus privilegierten Gruppen, gegenüber den Kindern der Bourgeoisie umso grundlegender sein: zumal die Studenten den Arbeitern nur in ihrer Eigenschaft als technische Spezialisten gegenübertreten konnten ohne im Zusammenhang eines wirksamen organisatorischen und politischen Rahmens zu stehen, in dem kollektives Handeln hätte sinnvoll sein können (34).

Es erwies sich als falsch, durch die Vermittlung von "systemtranszendierenden Bedurrfni sinterpretationen" einen Prozeß "revolutionärer Transformation" initiieren zu wollen, wie Offe dies als Funktion der "professionalisierten Intelligenz" konzipiert hatte. Es zeigte sich, daß die Arbeiter sich nicht von Bildern einer "konkreten Utopie" in spontanen Aktionsgruppen mobilisieren lassen; daß ihnen klar ist, daß diese winschenswerten Utopien nicht etwa nur deshalb noch nicht Realitàt geworden sind, weil sie es noch nicht zur kollektiven Artikulation solcher Bedürfnisse gebracht haben. Vielmehr nehmen sie deren Realisierung 
entgegenstehenden Widerstände viel umfassender wahr, als es eine Theorie wahrhaben will, die in diesen Widerständen "Sachgesetzlichkeiten" sehen zu können glaubt, die "prinzipiell auflosbar, nur jeweils noch nicht aurgelöst sind". (35) Wenn es aber nicht gelingt, fur den $p$ la n e $\mathrm{i}$ s ch dargestellten Konflik? zwischen "kollektiver Deprivation der Wirklichkeit" und "anschaulicher Msglichkei it" die von diesem Konflikt am stärksten Betroffenen zu mobilisieren; und wenn nicht die durch Konfliktdrohung einer "wiederbelebten Öffentlichkeit" zurückzudrängenden "Sachzwänge" sondern vielmehr der Kapitalverwertungszwang den Entscheidungsspielraum der Planungsagenturen limitiert, zerbricht der Wirkungszusammenhang, den Offe schematisch in dem Modell der planungsbezogenen Basisorganisation konstruiert hat. Da das vorgeblich Einheit stifende Moment einer allenthalben aktualisierbaren Stcatsintervention zur Vermeidung politischer Krisen sich als illusionär erweist, lassen die konfligierenden Interessen in der Realitöt des Planungsprozesses der professionalisierten Intelligenz nur noch die Alternative: Perfektionierung technokrotischer Verfohrensweisen durch ein formaldemokratisches Alibi oder Parteinahme für das Proletariat im Antagonismus von Diktafur der Bourgeoisie und Diktatur des Proletariats.

Die Basisgruppe Sanierungsgebiet Kreuzberg zog aus dem Scheitern der beschränkten Aktionsstrategie die Konsequenz, den Klassencharakter der Sanierungsprogrammarik in den Vordergrund der Agitation zu rücken. Damit geriet die politische Argumentation in offenen Widerspruch zur organisatorischen Basis, was ein Teil der Gruppe zu lösen suchte durch die Weiterfuhrung der Stadtreilarbeit im Rahmen einer Organisation mit Parteianspruch. Das Stadtteilkomitee der KPD/ML griff die Einstellung des Krankenhausbetriebes von Bethonien ouf und stellie sie in der Agi tation in den Zusammenhang der Verschlechterung der Krankenversorgung der Arbeiter, die dem Interesse des kapitalistischen Staates an der Minimierung der Reproduktionskosten der Arbeitskraft entspringt. Im Verlauf der Bethanien-Kampagne gelang espotentielle Arbeiterkader für diese Organisation zu gewinnen. Die Tarsche, daß eine größere Anzahl von Arbeitern mobilisiert wurde, zeigt, daß nicht die vielbeschworene Apathie von bewuBtlosen Verwaltungsobjekten der Grund fur den FehIschlag beschrönkterer Initiativen ist, sondern daß im Gegenteil gerade diese Beschränkung Abwehr bei den Arbeitern hervorruft.

Als Antwort darcuf daß die Agitation im Sanierungsgebiet in die Organisierung des proletarischen Klassenkampfes énbezogen wurde, ubernahm die Plonungsinstitution selber die Initiative für eine Strategie der Bürgerbeteiligung am Planungsprozeß, wie dies Offe ja auch empfohlen hatte. In Zusammenarbeit mit dem Kirchenparlament der Kreissynode Kölln-Stadt unterstutzte das Stadiplanungsami von Kreuzberg die Bildung eires Ausschusses fur Fragen der Stadrerneuerung unter Vorm sirz des Bezirksstadtrates für Wirtschaft, der gleichzei tig Sydonalvertreter einer Kreuzberger Gemeinde ist. Langfristiges Ziel der Gruppe ist die Konstituierung

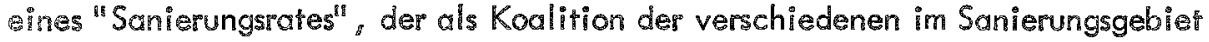
vertretenen Interessen sich an der Durchfuhrung der Sanierung beteiligen will. 
Vorerst versucht die Gruppe mit dem Gameindepforrer an ihrer Spitze, um den Kern des Ausschusses interessierte "professionalisierte Intelligenz" und "Mulhiplikatoren" aus dem Gebiet zu versammeln wie Lehrer, Kindergärtner innen , Kneipiers u.a. Um ihren Einfluß auf die entscheidenden lnstanzen zu verstarken, versucht die Gruppe, sich unentbehrlich zu machen als Kafalysator-oder wie der Pfarrer es nenni, als "ehrlicher Mokler" der die "aufgeklärre Kooperations= bereitschaffit der Arbeirerbevölkerung hersiellt. Zu diesem Zweck fuhrte die Gemeinde im November 1969 zusammen mit dem Planungscmt eine "Woche des Gesprächs" durch. Obwohl diese Gruppe, anders als kurzfristig auftretende studentische Basisgruppen, mit einem Vertrauen auf die Kontinuitat ihrer Arbeit rechnen konnte, vertrauten sich ihr die Arbeiter nicht an: Das Publikum beschrïnk te sich auf Planer, Bezirkspoliriker, "professionalisierte lnielligenz" und wenige Gemeinde-Omas. Von der Kreuzberger Arbeiterbevollkerung isoliert, hatte der Ausschuß nur eine Funktion als (allerdings recht nebensöchliches) Instrument zur Anpassung an das Unvermeidliche. So inszenierte der Ausschuß zu den Bauwochen 1970 eine autoritär manipulierte Bürgerbeteiligungs-Veranstaltung unter dem Theam "Sanierung Kreuzberg - unter Beteiligung der Óffentlichkeit"? Kernstuck der Veransialtung war ein Referat des Bauleitplaners vom Bezirksplanungsamt, in dem die Sanierungsplanung dargestellt wurde als ein Zusammenspiel von Verwaltung, parlamentarischer Öffentlichkeit und Interessengruppen, das mil der Verabschiedung des Flächennutzungsplanes im April 1970 durch das Abgeordnetenhaus bereits seinen unwiderruflichen gesetzlichen Abschluß gefunden habe. Es nimmt nicht wunder, daß dieser Nachhilfe-Unterrich in "adaquarer Wahrnehmung politischer Prozesse" (36) von den Kreuzberger Arbeitern nicht in Anspruch genommen wurde. Das Publikum, vor dem diese Ausführungen gemacht wurde, bestand wiederum nur aus einigen Rentnerinnen und einer diesmal noch großßeren Anzahl von fortschrittlichen Planern und Architekten, die ihr soziales En gagement hier in der "aufgeklärten Kooperation mit dem Institutionen" in Handeln umsetzen zu können hofften.

Auch in diesem Vorstoß auf etablierter Ebene wurde also deutlich, daß die Strow tegie, sich auf Konflikte der Disparität in einzelnen Lebensbereichen zu beschränken, für Arbeiter keine Handlungsperspektiven bieten konnte. Die Aktionsstrategie von "Situationsgruppen" durfte am shesten dem BewuBtseinsstand einer gehobenen Mittelschicht entsprechen, die Ausbeutung nicht unmitrelbar erfährt, fur die Disparihortskonflikte sich deshalb als skandalöse Ausnahmeerscheinungen darstellen können. So erwies sich des Bedurfnis, die eigene Wohngegend zu erhalien, z.B. im Frankfurter Westend, einem Wohmbezirk der Mirtelschicht, als durchaus konflikt-und organisationsfuhig. Die BurgerinitionivGruppe des Gebietes hatte ihr "Vertreuensverhältnis zur Stadt in Gefahr" gesehen. wenn es wirklich "der Stadt nicht möglich sei, sich gegen den im Westend austobenden Kapitalismus zur Wehr zu serzen" und wenn sich die Behorde woiterhin als unfähig arwiesen, an die prektischen Nöre der Westender zu denken. Im Unrerschied dozu ließ sich bei den im Sonierungsgebiet Kreuzbero wohnenden, zu- 
meist wenig qualifizierten Arbeitern und ihren Familien kein Vertrauen auf die Stad mobilisieren, dessen Enttäuschung Ansatzpunkt zur Politisierung und Selbstorganisation hätte bieten können.

Was wir uber die raclen Chancen der Durchserzbarkeit von Forderungen lokaler Gruppen in disparitåren Bereichen gesagr haben, gilt aber auch für das Westend: Trotz aller Beachinng, die den Westendern von Seiten kommunaler und parteipoliti scher Institutuionen widerfuhr -sogar der Bebaungsplan wurde geänderthaben sie praktisch keinerlei Durchsetzung ihrer Interessen erreicht. Der Marktmechanismus und auf Bundesebene erlassenen Wohnbaufinazierungsbestimmungen besorgen unerschütterlich die Verdrängung der jetztigen Bewohner. Eine politische Antwort auf die relative Verschlechterung der Wohnbedingungen der Lohnabhängigen, die natürlich a $\mathrm{ch}$ bewirkt wird durch die Umstrukturierung der Agglomerahionsräume nach den Erfordernissen der konzentrierten Kapitale, kann nicht bestehen in isolierten Kämpfen gegen die Erscheinungsformen dieser Kapitalstrategie $n$, wie dies in den hehandelten Beispielen von Stadtteilarbeit betrieben wurde. Wie die Geschichte der Wohnungsgesetzgebung der Bourgeoisie und ihrer Sozialgesetzgebung überhaupt zeigt, kann der Kampf gegen die Verschlechterung der Wohnbedingungen Erfolge überhaupt nur aufweisen, wenn er gefuhr wird im Zusammenhang des organisierten Kampfes der Arbeiterklasse gegen die Bourgeoisie.

\section{ANMERKUNGEN}

1 Vgl. Joachim Bergmann, Gerhard Brandt, Klaus Körber, Ernst Theodor Mohl, Claus Offe: "Herrschaft, Klassenverhältnis und Schichtung", Referat auf dem 16. Deutschen Soziologentag, Frankfurt 1968 , Manuskriptdruck sowie in: SPÄTKAPITALISMUS ODER INDUSTRIEGESELLSCHAFT ? Stuttgart 1969, S. $67 \mathrm{ff} . \mathrm{Vgl}$. auch Claus Offe: "Politische Herrschaft und Klassenstrukturen. Zur Analyse spätkapitalistischer Gesellschaftssysteme", in: POLITIKWISSEN SCHAFT. Eine Einführung in ihre Probleme. Hg. von Gisela Kress und Dieter Senghaas. Frankfurt/Main 1969, S. 155 ff. Diese Arbeiten bzw. Vorarbeiten dazu sind ausserdem in zahlreichen Privatdrucken verbreitet.

Vorsichtige Rücknahme früherer Positionen ine Claus Offe: "Notitzen zum Thema -Planung im Beruf-"1". In: Stadtbauwelt 61. 1970. H. 28, S. 277 - 279. Es geht hier um die Korrekfur einer falschen Konzeption von Stedtreilarbeit, die von Offe theoretisch ausformuliert wurde, nicht um eine Auseinanderselzung mit Offe als Person. Aus diesem Grunde beschäfrigen wir uns hier nicht mit dem zuletzł genannien Aufsatz. 
2 z.B. Interview mit Karsten Voigt, Juso-Vorsitzender, mit der Frankfurter Rundschau 23.9.1970

Wichrig warre in diesem Zusammenhang eine Untersuchung der Stadtteilarbeit von DKP-Gruppen (2.B. im Munchener Lehel), der objektiv - allem Anschein nach wenigstens - die gleichen Tendenzen zugrunde liegen, was insbesondere in einem ungeklärten Verhältnis von Stadtteilarbeir und Betriebsarbeit zum Ausdruck kommt.

3 Offe, ..a.O., S. $180 \mathrm{f}$.

4 Ebda.,5. 183

5 Ebda., S. 185

6 Ebda., S. 175

7 Bergmann $u . a_{0}, a . a .0 ., 5.87$

8 Claus Offe: "Sachzwang und Entscheidungsspielraum" in: STADTBAUWELT, Nr. 38/39, Berlin 1969, 5. $187 \mathrm{ff}$.

9 Ebda., 5. 190

10 Ebda., 5. 191

11 Ebda., S. 189

12 Ebda.

13 Ebda., S. 191

14 Paul Davidoff: "Advocacy and pluralism in planings" in: JOURNAL OF THE AMERICAN INSTITUTE OF PLANERS, Nov. 1965, S. $331 \mathrm{ff}$.

15 Claus Offe: "Sachzwang und Entscheidungsspielraum", $0 . a .0 ., 5.191$

16 Ebda., (Sperrung H.F.)

17 Vgl. im folgenden Danny Beagle, Al Haber, David Wellman: "Turf power and the man. Urban renewal, regionalization and the limits of community conirol", in: LEVIATHAN, Nr. 2, April 1969. Deutsch in: SANIERUNG FŮR WEN ? a.a.O., S. $123 \mathrm{ff}$.

18 Ebda., S. 131. Wir erkennen hinter dieser scheinbar rein verwaltungstechnischen "Modernisierung" eine interessante Entwicklung. Während in der Zeit der offenen politischen Herrschaft der Bourgeoisie das Wahlrecht nach Vermögen gestaffelt war (Zensus-Wahlrecht), wurde angesichts des drohenden Anwachsens der Arbeiterbewegung das allgemeine Wahlrecht zugestanden, welches eine Basis der politischen Integration der Arbeiter als "Staatsbuirger" in die "demokratische Gesellschaft" war. Heute scheint der Nebelschleier der "Sachgesetzlichkeit", der "Effizienz" u.s.w. für die großkapitalistische Bourgeoisie die parlamentarische Bemäntelung ihrer Klassenherrschaft ubberflüssig zu machen, zumindest auf den unteren und mittleren Ebenen der politischen Entscheidungen. Wenn sich Claus Offe schon die Mühe machì, die für die Erhaltung spätkapitalistischer Herschaftssysteme problematischen Folgen des "Austrocknenes" der Institutionen der Öffentlichkeir nachzuweisen, so härte er auch einmal danach fragen sollen, in wessen Interesse diese Institutionen trockengelegt werden. 
19 Die direkte Abhängigkeit der Linienfuhrung der verschiedenen Verkehrssysteme von den Bedürfnissen der stärksten Kapitale läßt sich z. B. auch an der Entstehung der S-Bahn und dem Ausbau der Wasserstraßen in Großberlin nøchweisen. Vgl. Horant Fassbinder, U.a.: BERLINER ARBEITERVIERTEL I. Von der ursprunglichen Akkumulation zum Monopolkapirelismus, Berlin 2. Auflage 1971

20 Nach einem Bericht des NATIONAL GUARDIAN v. 27.5.67 betrug die Arbeitslosigkeit unter der Ghetrobevolkerung von 13 US-amerikanischen Großstödren durchschnittlich 10\%. Die Rate von "Unterbeschäftigung" (Arbeitslose, "Arbeitsunwillige" Unterbezchltes, deren Einkommen unter der offiziellen Armursgrenze von 3300 Dollar jărlich fur eine vierköpfige Familie liegt) betrug durchschnithlich $33,9 \%$ in San Francisco (MissionFillmore) betrug die Arbeitslosenquote $11,1 \%$ die der Unterbezahiten 24,6\%. Aus: NOW. DER SCHWARZE AUFSTAND, hg. $v . V . U$. Brandes, Munchen 1968, S. 105

21 Beagle U.a., 0.0.0., \$. 135

22 Ebda.

23 Ebold.. S. 124

24 Ebda., S. 136 f。

25 Ebda., S. 137

26 Ebda., S. 138

27 Ebda.

$28 \mathrm{Vgl}$. dazu die Lokalteile der SUDDDEUTSCHEN ZEITUNG u.d.Frankf. Runds.

29 Zu geringer Warenumsatz pro Quadratmeter oder pro Beschäftigten wird in den Sanierungsberichten des Berliner Senats håufig als Kriterium der Sanie. rungswirdigkeit angeführt.

30 Der Abend v. 3. 12. 70 berichtet daruber: "Für über 73 Mio. Mark soll nördlich des Kortbusser Tores das "Neue Kreuzberger Zentrum" entstehen. Für diese Randbebauung, die im Sanierungsgebiet des Bezirks liegt, wurden zuvor strukturelle und soziologische Erhebungen angestellt. Das Ergebnis ist ein siodrebaulich reizvolles "Mischprojek", das bis 1974 verwirklicht sein soll ... Angrenzend an die Skalitzer/Gitschiner Straße sollen -z.T. in Hochciusern- 300 familiengerechte Wohnungen, ferner $15000 \mathrm{gm}$ Gewerbeflache als "Beletage der Kreuzberger Industrie geschaffen werden. Neben zahlreichen Geschäften und möglicherweise auch zwei Kaufhäusem wird as auch eine Reihe von Sozialeinrichrungen geben. Mittelpunkt mit Forumcharakter bildet ein Lesehof im Anschluß an die schon bestehenden Srädr. Bucherei, der auch als Freilichttheater genutzt werden kann. Dem Hochhous Kern wird ein gärtnerisch gestalteter "grüner Ring" umziehen. Für 700 Autos ist in Parkhäusern Platz. Für das geplante Wohn und Geschåftsvier w tel mußten 29 Grundstücke erworben werden. Seine besondere Note erhält der Komplex durch einen Bruckenbau , der die Adalbertstraße úberspannt . 
Durch die später hier entlangfuhrende Stadtautabahn-Tangente besteht ein minutenschneller Anschluß an die City".

Auf die Schwierigkeiten, die alten Eigentümer zum Verkouf zu bewegen, ohne daß diese bereits jetzł große Teile der antizipierten Grundrente in kapitalisierter Form einstreichen, kann hier nur hingewiesen werden. Seit ca. 1963 bereits ziehen sich die Versuche des großen Industrie- und Handelskapitals hin, dies Hindernis schneller und vor allem billiger Sanierung zu beseitigen durch ein Städrebauförderungsgesetz.

31 Stadrerneuerung in Berlin. Erster Bericht an das Abgeordnetenhaus von Berlin. Drucksachen des Abgeordnetenhauses von Berlin. Nr. 556, 12.6.1964 S. 3.

32 Der Begriff "Infrostruktur" wird heir nicht im bescheänkt technischen, sondern im polit-ökonomischen Sinne gebraucht: Als die Gesamtheit jener staatlichen Vorleistungen für die private Akkumulation, deren Planung, Finanzierung und Nutzung die Bourgeoisie als gemeinschaftliches Geschäft betreibt aus jenem Teil des Mehrwerts, der in der Form von Stevern an ihren sraatlichen Apparat fällt. Dafür, ob etwas der Infrastruktur zugerechnet wird oder nicht gibt es nicht die eindeutigen technischen Kriterien, nach denen bürgerliche Nationalökonomen vergeblich suchen. Das Interesse der mächtigsten Fraktionen der Bourgeoisie - oft genug erst erzwungen durch den Kampf der Arbeiterklasse - ist das einzige Kriterium dafur, ob eine Investition gemeinschafflich durchgefuhrt wird oder individuell. Naturlich geht weder die Aufbringung dieses Teils des Mehrwerts noch seine Verwendung ab ohne "höchst erbaulichen Strei î" zwischen den verschiedensten Fraktionen der Bourgeoisie.

Fur Berlin werden die Ursachen für die Veränderung der Infrastruktur fur die Zeit bis zum Ersten Weltkrieg untersucht in: Horant Fassbinder u.a.: BERLINER ARBEITERV?ERTEL 1. Von der ursprünglichen Akkumulation zum Monopolkapiralismus, Berlin 197]

33 Siehe dazu: Horant Fassbinder: Die Stellung der Industrie- und Handelskammer Berlin zur Berliner Stadisanierung anhand ihrer Jahresberichte 1960 . 1959. Unveroffentl. Manuskript, 1970

34 Die individuelle Erichrung der eigenen Ohmocht "denen da oben" gegenuber zusserte sich in den Untersuchungsgesprochen hiufig in Sätzen wie: "Die de oben mochen ja doch, wos sie wollen" ode" "Die geben uns wos, die nehmen uns wes". Diese Erfehrung schligh cul he Bereischent zur Op ganisat ion und aut die Einscharzung dep potentiellen Orgenisierung yon

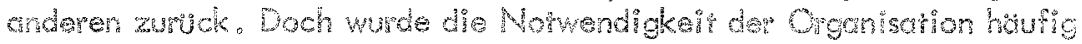

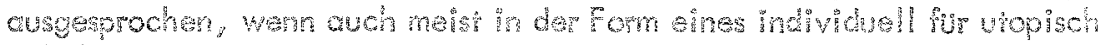
geholsenen Wuntohes. 
35 Claus Offe: "Sachzwang und Entscheidungsspielrourn" , a,a, O, S. 191

36 Derselbe Bauleitplaner erklarte in einem informellen Gruppengesprdch, ein "Zirkulieren von Unterprivilegierten mit nachfolgenden Slumerscheinungen", wie es sich in amerikanischen und westeuropaischon Großstadten als Folge der Flachensanierung gezeigit habe, werde sich auch in Westberlin nicht vermeiden lassen.

\section{MATERIALIEN \\ DM 11,50}

\section{ZUR POLITISCHEN ÖKONOMIE DES \\ AUSBILDUNGSSEKTORS}

Herausgegeben von

Elmar Altvater und Freerk Huisken

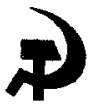

\section{Erlangen 1971}

\section{Bestellung von Einzelexemplaren}

ist möglich über die Buchläden des Verbandes des linken Buchhandels oder direkt beim Veriag POLITLADEN GmbH, Erlangen. 